\title{
Total knee arthroplasty in the next decade: is navigation necessary?
}

\author{
Graham S. Goh, Ming Han Lincoln Liow
}

Department of Orthopaedic Surgery, Singapore General Hospital, Singapore, Singapore

Correspondence to: Ming Han Lincoln Liow, MBBS, MRCS (Edin), MMed (Ortho), FRCSEd (Orth), FAMS. Department of Orthopaedic Surgery, Singapore General Hospital, 20 College Road, Academia, Level 4, Singapore 169865, Singapore. Email: dr.lincoln.liow@gmail.com.

Provenance and Peer Review: This article was commissioned by the editorial office, Annals of Translational Medicine. The article did not undergo external peer review.

Comment on: Xu X, Liu P, Yuan Z, et al. Comparison of a novel handheld accelerometer-based navigation system and conventional instrument for performing distal femoral resection in total knee arthroplasty: a randomized controlled trial. Ann Transl Med 2019;7:659.

Submitted Feb 03, 2020. Accepted for publication Mar 06, 2020.

doi: 10.21037/atm.2020.03.183

View this article at: http://dx.doi.org/10.21037/atm.2020.03.183

The optimal alignment goal in total knee arthroplasty (TKA) has become a topic of debate lately. Traditionally, surgeons have aimed for a mechanical axis of less than $3^{\circ}$ from neutral in order to prevent early failure, especially on the tibial side (1). However, some authors have challenged this belief, reporting no difference in the 15 -year survivorship between mechanically aligned TKAs and outliers with a mechanical axis of greater than $3^{\circ}$ from neutral (2). More recently, proponents of a newer alignment method known as "kinematic alignment" have also questioned the concept of mechanical alignment, citing similar or better clinical outcomes at short-term follow-up (3), although studies comparing the long-term survivorship of the two methods are lacking.

While the optimal alignment goal in TKA has not been determined, surgical accuracy and reliability in achieving a desired alignment target remains a priority for surgeons. Rapid technological advancements have led to the development of a variety of navigation systems, including image-based or imageless computer-assisted navigation systems (CAS) and patient-specific instrumentation (PSI). However, despite the superior accuracy of this technology (4), a meta-analysis identified very few clinical advantages of CAS (5). Furthermore, several disadvantages exist, such as the learning curve required, immense capital expenditure, increased surgical duration, pin site complications and lineof-sight problems (5). These disadvantages have limited the widespread adoption of CAS techniques.

To address some of these drawbacks, handheld accelerometer-based navigation $(\mathrm{ABN})$ was recently introduced, with the two major systems on the market being the KneeAlign2 (OrthAlign, Aliso Viejo, CA) and iASSIST (Zimmer, Warsaw, IN). ABN aims to harness the accuracy and precision in CAS while maintaining the familiarity of conventional instrumentation (CON) (6). The KneeAlign2 consists of a display console, reference sensor and attachments to tibial and femoral jigs. The console communicates wirelessly with the reference sensor to guide femoral and tibial resection in the sagittal and coronal planes. Similarly, the iASSIST uses multiple accelerometer or gyroscopic components that relay information to each other wirelessly using Wi-Fi. Information on the alignment is then shown to the surgeon on the user interface, directly within the surgical field. Following which, the data can be used to guide the tibial and femoral resection. The workflow of ABN not only approximates conventional methods, but also provides digital feedback and anatomical landmark referencing for surgeons (7). Advantages of these systems also include a gentler learning curve, minimal capital expenditure, lack of pin-related complications, added convenience with no need for other devices outside the surgical field and/or lack of line-of-sight issues, and open platform software that can be used with different prostheses (6). Despite these advantages, inherent limitations of accelerometer-based technology include the lack of information on rotational alignment of the femoral and/or tibia components [another key determinant of long-term survivorship and functional outcomes (8)], the inability to 
assess soft tissue balancing, as well as the increased cost of disposable single-use electronic pods.

In a recent issue of the Annals of Translational Medicine, $\mathrm{Xu}$ et al. conducted a double-blind, prospective randomized study investigating the accuracy of a new ABN system known as the i-JOIN knee navigation system (i-JOIN Medical Technology, Shanghai) (9). A total of 39 patients had the femoral resection performed with the use of the i-JOIN ABN device, while 40 patients underwent surgery using a conventional intramedullary guide. Both groups had tibial resections performed using conventional extramedullary guides, hence ABN-TKAs were only "partially" navigated in this study. The authors observed that the intraoperative blood loss and rate of perioperative adverse events were similar between the groups. However, there was no significant difference in hip-knee angle (HKA), coronal femoral angle (CFA) or the percentage of outliers with mechanical axis $>3^{\circ}$ from neutral, despite a significantly longer operative time in the $\mathrm{ABN}$ group. The follow-up duration was only one week, hence functional outcomes were not analyzed or compared.

In contrast with the findings of $\mathrm{Xu}$ et al., the majority of studies have reported that the use of $\mathrm{ABN}$ compared to $\mathrm{CON}$ resulted in a significant reduction in outliers for the HKA (10-14) and CFA (12,15-17), although improved accuracy in the coronal tibial angle (CTA) was less commonly demonstrated $(12,17-21)$. Only one study by Fujimoto et al. found a significant reduction in CTA outliers in the ABN group (11). Furthermore, improved alignment accuracy in the sagittal plane was difficult to demonstrate, as few studies found a reduction in outliers for the sagittal tibial angle (STA) and sagittal femoral angle (SFA) (11,15,17-21). The accuracy of ABN has also been compared with the accuracy of newer technology like CAS (22-24) and PSI (15,25). Goh et al. conducted a prospective matched cohort study on 38 iASSIST TKAs versus 38 CAS TKAs and found comparable postoperative alignment measurements between the groups (22). Nam et al. even proposed that the KneeAlign2 was more accurate than the CAS as they observed a reduction of outliers for HKA and CFA (23). When comparing ABN and PSI, one study did not find a difference in coronal alignment or the proportion of outliers with $\mathrm{a}>3^{\circ}$ deviation from neutral (25), whereas another found significantly fewer outliers in the HKA, CFA and SFA in the ABN group compared to the PSI group (15). Current evidence suggests that the accuracy of $A B N$ is at least comparable to that of CAS and PSI with the added benefit of a shorter operative time $(22,23)$.
As a more neutral mechanical alignment may lead to better survivorship (1), one may naturally infer that the use of CAS, $A B N$ or other navigation techniques could therefore lead to decreased revision rates. However, this has not been proven in previous studies (26). Improved patientreported outcomes with the use of navigation devices has also been difficult to demonstrate (5). After prospectively matching 152 patients for age, gender, BMI, preoperative range of motion, clinical scores and preoperative coronal alignment, Goh et al. compared CAS, ABN and CON-TKA at a follow-up of 2 years (12). Despite the superior precision and accuracy of $\mathrm{ABN}$ and CAS in achieving a neutral $\pm 3^{\circ}$ mechanical axis after TKA, the authors could not find any difference in clinical outcomes or patient satisfaction. These findings were also shared by other studies with shorter follow-ups $(13,17)$. Additional studies with longer followup are required to determine if the improved alignment in $\mathrm{ABN}$-TKA results in improved functional outcomes for patients.

The increased operative time with the use of new technology is another area of concern. Although the learning curve of ABN-TKA has not been evaluated thoroughly, several studies have compared the operative time for ABN-TKA and CON-TKA $(10,12,15,17,18,20)$. When considering the time from skin incision to wound closure, Liow et al. found that the ABN group had a significantly increased operative time $(84 v s .73 \mathrm{~min}$ in CON group) (13). In contrast, despite the heterogeneity of surgical factors such as operative experience and learning curve, the majority of studies in the literature did not find a difference in operative duration $(10,12,15,17,18,20)$, which may be attributed to the minor adjustment in surgical technique. Among the few studies analyzing tourniquet time $(14,16)$, none found a significant difference between the $\mathrm{ABN}$ and $\mathrm{CON}$ groups as well. $\mathrm{ABN}$ provides a level of familiarity to surgeons as its electronic pods attach onto conventional resection instruments, making the surgical workflow approximately the same as the conventional technique. Using the handheld navigation device directly within the operative field bypasses the inconvenience of having large consoles in the operating room and line-ofsight issues with optical navigation.

As healthcare systems transition towards value-based care, another major concern with new technology is the additional costs incurred with its implementation. It is estimated that the use of CAS increases the cost of TKA by $\$ 1,500$ per case, although it has also been suggested that CAS could potentially be cost-effective in the long run 
if the improved consistency of alignment translated to a decrease in revision rates (27). Although $\mathrm{ABN}$ increases the cost per procedure, it avoids the sizable costs of purchasing and servicing a CAS console. Goh et al. recently compared the costs of iASSIST-TKA and CAS-TKA, estimating the cost of $A B N$ to be $\$ 1,000$ higher per case (22). However, registry data on the cost-effectiveness of $\mathrm{ABN}$ are lacking, hence it remains uncertain whether the improved alignment using $\mathrm{ABN}$ will translate to reduced revision rates and greater cost savings for patients.

In current orthopaedic practice, surgeons considering the use of navigation tools are often faced with three main questions: (I) what is the ideal alignment we should aim for in TKA? (II) What is the best method to achieve it? (III) Can achieving this lead to clinical benefits for the patient? While the literature suggests that CAS and ABN can improve the accuracy in achieving a desired alignment target in TKA, albeit at an increased cost per operation, the first question on the optimal alignment goal remains unanswered. ABN may provide an additional benefit of familiarity for surgeons accustomed to CON, leading to shorter surgical times compared to other navigation techniques. However, like its predecessor, large-console CAS, the introduction of handheld ABN may not necessarily translate to clinical advantages such as better functional outcomes or implant survivorship. It is plausible that the reason why the clinical benefits of new navigation techniques have not come to fruition is because our current alignment goal may not be the optimal one. In addition to investigating the long-term outcomes of $\mathrm{ABN}$ in large registry-based studies, surgeons should also tackle the fundamental question on whether postoperative alignment is a key determinant of clinical outcomes after TKA. Similar to how global positioning systems (GPS) guide our vehicles to the correct destination, navigation devices have a role to play in TKA, but we need to know where to go in order to harness the full potential of this new technology.

\section{Acknowledgments}

We would like to thank Ms. Sarah Boey for helping us to proofread and format this manuscript.

Funding: None.

\section{Footnote}

Conflicts of Interest: Both authors have completed the
ICMJE uniform disclosure form (available at http://dx.doi. org/10.21037/atm.2020.03.183). The authors have no conflicts of interest to declare.

Ethical Statement: The authors are accountable for all aspects of the work in ensuring that questions related to the accuracy or integrity of any part of the work are appropriately investigated and resolved.

Open Access Statement: This is an Open Access article distributed in accordance with the Creative Commons Attribution-NonCommercial-NoDerivs 4.0 International License (CC BY-NC-ND 4.0), which permits the noncommercial replication and distribution of the article with the strict proviso that no changes or edits are made and the original work is properly cited (including links to both the formal publication through the relevant DOI and the license). See: https://creativecommons.org/licenses/by-nc-nd/4.0/.

\section{References}

1. Berend ME, Ritter MA, Meding JB, et al. The Chetranjan Ranawat Award: Tibial Component Failure Mechanisms in Total Knee Arthroplasty. Clin Orthop 2004;428:26-34.

2. Parratte S, Pagnano MW, Trousdale RT, et al. Effect of Postoperative Mechanical Axis Alignment on the Fifteen-Year Survival of Modern, Cemented Total Knee Replacements: J Bone Joint Surg Am 2010;92:2143-9.

3. Courtney PM, Lee GC. Early Outcomes of Kinematic Alignment in Primary Total Knee Arthroplasty: A MetaAnalysis of the Literature. J Arthroplasty 2017;32:20282032.e1.

4. Mason JB, Fehring TK, Estok R, et al. Meta-Analysis of Alignment Outcomes in Computer-Assisted Total Knee Arthroplasty Surgery. J Arthroplasty 2007;22:1097-106.

5. Bauwens K, Matthes G, Wich M, et al. Navigated Total Knee Replacement. J Bone Joint Surg Am 2007;89:261-9.

6. Jones CW, Jerabek SA. Current Role of Computer Navigation in Total Knee Arthroplasty. J Arthroplasty 2018;33:1989-93.

7. Scuderi GR, Fallaha M, Masse V, et al. Total Knee Arthroplasty with a Novel Navigation System Within the Surgical Field. Orthop Clin North Am 2014;45:167-73.

8. Bell SW, Young P, Drury C, et al. Component rotational alignment in unexplained painful primary total knee arthroplasty. Knee 2014;21:272-7.

9. Xu X, Liu P, Yuan Z, et al. Comparison of a novel handheld accelerometer-based navigation system and 
conventional instrument for performing distal femoral resection in total knee arthroplasty: a randomized controlled trial. Ann Transl Med 2019;7:659-9.

10. Lo CK, Li HY, Wong YC, et al. Total Knee Replacement with iASSIST Navigation System. J Orthop Trauma Rehabil 2018;24:29-33.

11. Fujimoto E, Sasashige Y, Nakata K, et al. Technical Considerations and Accuracy Improvement of Accelerometer-Based Portable Computer Navigation for Performing Distal Femoral Resection in Total Knee Arthroplasty. J Arthroplasty 2017;32:53-60.

12. Goh GSH, Liow MHL, Tay DKJ, et al. AccelerometerBased and Computer-Assisted Navigation in Total Knee Arthroplasty: A Reduction in Mechanical Axis Outliers Does Not Lead to Improvement in Functional Outcomes or Quality of Life When Compared to Conventional Total Knee Arthroplasty. J Arthroplasty 2018;33:379-85.

13. Liow MHL, Goh GSH, Pang HN, et al. Computerassisted stereotaxic navigation improves the accuracy of mechanical alignment and component positioning in total knee arthroplasty. Arch Orthop Trauma Surg 2016;136:1173-80.

14. Kinney MC, Cidambi KR, Severns DL, et al. Comparison of the iAssist Handheld Guidance System to Conventional Instruments for Mechanical Axis Restoration in Total Knee Arthroplasty. J Arthroplasty 2018;33:61-6.

15. Kawaguchi K, Michishita K, Manabe T, et al. Comparison of an Accelerometer-Based Portable Navigation System, Patient-Specific Instrumentation, and Conventional Instrumentation for Femoral Alignment in Total Knee Arthroplasty. Knee Surg Relat Res 2017;29:269-75.

16. Ikawa T, Takemura S, Kim M, et al. Usefulness of an accelerometer-based portable navigation system in total knee arthroplasty. Bone Joint J 2017;99-B:1047-52.

17. Ueyama H, Matsui Y, Minoda Y, et al. Using Accelerometer-Based Portable Navigation to Perform Accurate Total Knee Arthroplasty Bone Resection in Asian Patients. Orthopedics 2017;40:e465-72.

18. Gharaibeh MA, Solayar GN, Harris IA, et al. Accelerometer-Based, Portable Navigation (KneeAlign) vs Conventional Instrumentation for Total Knee

Cite this article as: Goh GS, Liow MHL. Total knee arthroplasty in the next decade: is navigation necessary? Ann Transl Med 2020;8(11):665. doi: 10.21037/atm.2020.03.183
Arthroplasty: A Prospective Randomized Comparative Trial. J Arthroplasty 2017;32:777-82.

19. Moo IH, Chen JYQ, Chau DHH, et al. Similar radiological results with accelerometer-based navigation versus conventional technique in total knee arthroplasty. J Orthop Surg (Hong Kong) 2018;26:2309499018772374.

20. Thiengwittayaporn S, Fusakul Y, Kangkano N, et al. Hand-held navigation may improve accuracy in minimally invasive total knee arthroplasty: a prospective randomized controlled trial. Int Orthop 2016;40:51-7.

21. Matsumoto K, Fukuta M, Mori N, et al. Comparative Study for Alignment of Extramedullary Guides versus Portable, Accelerometer-Based Navigation in Total Knee Arthroplasty. J Knee Surg 2018;31:92-8.

22. Goh GSH, Liow MHL, Lim WSR, et al. AccelerometerBased Navigation Is as Accurate as Optical Computer Navigation in Restoring the Joint Line and Mechanical Axis After Total Knee Arthroplasty. J Arthroplasty 2016;31:92-7.

23. Nam D, Weeks KD, Reinhardt KR, et al. AccelerometerBased, Portable Navigation vs Imageless, Large-Console Computer-Assisted Navigation in Total Knee Arthroplasty. J Arthroplasty 2013;28:255-61.

24. Desseaux A, Graf P, Dubrana F, et al. Radiographic outcomes in the coronal plane with iASSISTTM versus optical navigation for total knee arthroplasty: A preliminary case-control study. Orthop Traumatol Surg Res 2016;102:363-8.

25. Steinhaus ME, McLawhorn AS, Richardson SS, et al. Handheld Navigation Device and Patient-Specific Cutting Guides Result in Similar Coronal Alignment for Primary Total Knee Arthroplasty: a Retrospective Matched Cohort Study. HSS J 2016;12:224-34.

26. Kim YH, Park JW, Kim JS. The Clinical Outcome of Computer-Navigated Compared with Conventional Knee Arthroplasty in the Same Patients: A Prospective, Randomized, Double-Blind, Long-Term Study. J Bone Joint Surg Am 2017;99:989-96.

27. Novak EJ, Silverstein MD, Bozic KJ. The CostEffectiveness of Computer-Assisted Navigation in Total Knee Arthroplasty. J Bone Joint Surg Am 2007;89:2389-97. 